\section{Empredimientos en tiempos de pandemia}

Entrepreneurship in times of pandemic

\section{Luis Fernando Lema*}

\section{RESUMEN}

En febrero del 2021 en el Ecuador muchos negocios y empresas quebraron; según la OIT 84.38I personas perdieron su empleo adecuado; en este sentido el objetivo de este artículo es visualizar el entorno mercantil actual, las oportunidades de desarrollo que podría tener el emprendedor y observar ideas de emprendimientos adaptados en pandemia con una perspectiva diferente; basándose en negocios existentes o nuevos que han sabido superar y adaptarse a la crisis. Partiendo de la revisión bibliográfica de conceptos, definiciones, modelos y tendencias; con un método deductivo, en base a las necesidades ya existentes por pandemia.

Palabras clave: Emprendimiento, economía, sector productivo, desarrollo económico, tasa de desempleo, pandemia Covid 19
*Master, Gerente Comercial Proalid, Quito, Ecuador, luisfernando@gmail.com, https://orcid.org/0000-0002-6630-6825

\section{REVISTA TECNOLÓGICA ciencia y educación Edwards Deming}

ISSN: 2600-5867

Atribución/Reconocimiento-NoCo mercial- Compartirlgual 4.0 Licencia Pública Internacional - CC

\section{BY-NC-SA 4.0}

https://creativecommons.org/licenses /by-nc- sa/4.0/legalcode.es

Editado por: Tecnológico Superior Corporativo Edwards Deming

Enero - Julio Vol. 5 - I - 202 I

https://revista-edwardsdeming.com/index.php/es e-ISSN: 2576-097I

Recibido: 04 Marzo 2020

Aprobado: II Diciembre, 2020

Pag 65-80 


\begin{abstract}
In February 2021 in Ecuador many businesses and companies went bankrupt; according to the OIT 84,38I people lost their proper employment; in this sense the objective of this article is to visualize the current business environment, the development opportunities that the entrepreneur could have and observe ideas of entrepreneurship adapted in pandemic with a different perspective; based on existing or new businesses that have been able to overcome and adapt to the crisis. Starting from the bibliographic review of concepts, definitions, models and trends; with a deductive method, based on the existing needs for pandemic.
\end{abstract}

Keywords: Entrepreneurship, economy, productive sector, economic development, unemployment rate, Covid 19 pandemic

\title{
INTRODUCCIÓN
}

La pandemia del Covid 19 sacudió al mundo; la economía global se desmorono, las empresas tuvieron que cerrar se dejó a muchas personas desempleadas las mismas que comenzaron a buscar la manera de icómo sobrevivir económicamente?; se crearon por necesidad emprendimientos no adecuados y con desorden, sumado a la iniciativa e ingenio cambiaron la forma de presentar los negocios.

Aplicando un enfoque interpretativo, el presente artículo procura demostrar que si se puede generar emprendimientos de manera apropiada con las nuevas medidas, restricciones y tendencias. Analiza fuentes escritas, videos contemporáneos, definiciones y conceptos para posteriormente dar ejemplos reales de nuevos y adaptados emprendimientos que mejoren la situación económica y podrían generar posteriormente fuentes de empleo en la pandemia y en la nueva realidad post pandemia.

\section{MATERIALES Y MÉTODOS}

Se utilizó el análisis cualitativo, contemporáneo sobre el efecto de la pandemia en el emprendimiento. Tiene una perspectiva aclarativa deductiva, siendo un trabajo conceptual y teórico.

Se buscó entender las nuevas tendencias de emprendimientos y la adaptación de los existentes a la nueva realidad partiendo de referencias e interpretando resultados.

Se tomó como base publicaciones actuales sobre negocios estables, en crecimiento y tendencias de consumo, para combinarles y presentar como posibles proyectos de negocios.

\section{RESULTADOS}

Emprender en un mundo globalizado es cuestión de actitud positiva, de un pensamiento crítico con valor para asumir riesgos según Parra Alviz, Rubio Guerrero, y López Posada (2019); para Real Academia Española (s.f.) la palabra emprender es "acometer y comenzar, un negocio, un empeño, especialmente si encierra dificultad o peligro." 
El emprendimiento es una herramienta y no la solución económica a la crisis de generación de empleo; las crisis económicas y la dificultad económica y social dan como posible resultado menos personas involucradas en emprendimientos según Martínez, y Rubio Bañón, (2020)

Con un orden económico aparentemente incierto nadie se imaginó que una pandemia restructuraría el mundo, cambiara la realidad, se pondría a prueba, con un futuro todavía algo fortuito y con muchas variables e información no definida. Lo que antes era visible y se podía proyectar, ahora no sabemos cómo va a funcionar y lo único seguro es el cambio constante y el reacomodo de nuestro entorno económico y social especialmente de los países en vías de desarrollo.

"El funcionamiento eficiente de una economía implicara maximizar los niveles de producción y de bienestar social a partir de los recursos disponibles y las políticas llevadas a cabo". (Expósito, Fernández-Serrano y Velasco, 2017, p.I I9).

"Se proyecta que la economía mundial crecerá un $4 \%$ en 2021 después de la contracción del 4,3\% del año pasado, la cuarta recesión mundial más profunda en los últimos 150 años". (Banco mundial, 5 de enero 202I). Muchos negocios han cerrado y el índice de pobreza ha aumentado en América Latina; en Ecuador según Torres (27 de febrero de 2021 ) se sitúa en el $32 \%$ en el 2020. Los negocios y empresas que quebraron dejaron al mundo con mucha gente desempleada, en el Ecuador " $8438 \mathrm{I}$ personas perdieron su empleo adecuado en febrero de 202 I" según el diario Comercio del 22 de marzo de 202I. En el mismo que destaca que el sub empleo y el desempleo en mujeres creció al $7,3 \%$ casi duplicando al de los hombres.

Si a esto le aumentamos que según el ministerio de educación en Ecuador el periodo 2019 - 2020 se graduaron de bachilleres 302.354 jóvenes y la oferta de educación superior apenas cubre a I 13.072 según el boletín de prensa No 017 del 3 de marzo del 2020 del SENECYT. Se genera un conjunto de jóvenes sin estudios superiores que buscan empleo y las opciones cada vez son menores; muchos buscan restructurar sus deudas con los bancos, otros simplemente han bajado los brazos en espera de que esto cambie y encuentren un trabajo adecuado para poder pagar sus deudas. Este ejemplo es una razón más por lo que es muy necesario reactivar la economía y el sector productivo, en especial al emprendedor como fuente de trabajo y ayuda en el mejoramiento de los índices de pobreza.

De persistir los efectos negativos de la epidemia, los países deberán adoptar medidas tanto fiscales como monetarias y financieras tendientes a apoyar a los consumidores y a las pequeñas empresas que están siendo las más afectadas. Además, deben tenerse en cuenta los distintos mecanismos de financiamiento con que cuentan tanto el Banco Mundial como el Fondo monetario Internacional para hacer frente a la emergencia actual. (Clavellina Miller, 2020).

\section{Emprendedor y tendencias en tiempos de pandemia}

"Son personas naturales o jurídicas que persiguen un beneficio trabajando individual o colectivamente. Pueden ser definidos como individuos que innovan, identifican y crean 
oportunidades, desarrollan un proyecto y organizan los recursos necesarios para aprovecharlos" (Asamblea Nacional de la Republica de Ecuador, 2020).

Todo emprendedor sabe que debe estar expuesto constantemente al cambio la resiliencia como capacidad para superar adversidades; demos entender que ser emprendedor antes y después de la pandemia no ha cambiado, lo que ha cambiado son las circunstancias, las variables, el ambiente.

Podríamos también afirmar que el emprendedor es un agente de cambio, que invierte en busca de utilidades a riesgo y que por varios factores como la falta de cálculo e investigación del mercado podría perder y posiblemente quebrar su emprendimiento o por diferentes variables generar ingresos positivos a su economía

Según el reporte del GEM (Global Entrepreneurship Monitor) por sus siglas en ingles que fue elaborado por la escuela de negocios de la Espol (Espae), 37 de 150 emprendimientos consultados cerraron durante la pandemia. El 42, I\% de los cierres se produjo en Guayaquil; 31,6\% en Quito; 10,5\% en Cuenca; el 5,3\% en las ciudades de Ibarra, Daule y las naves. (IB Training Center, 3 de agosto de 2020).

Para los empresarios les dejó algunas lecciones, una sensación nada buena, por no haber invertido adecuadamente en productos relacionados con la pandemia; algunos se agotaron debido a la excesiva demanda llegando a faltar por meses, en especial las materias primas importadas y medicamentos; otros productos se quedaron sin vender, repletos en inventarios con productos elaborados; debido a su desuso o a la incorporación de productos sustitutos, como es el ejemplo de los trajes anti fluidos o las bombas para fumigar.

En 2019, la tasa de TEA (Actividad económica temprana) de Ecuador fue del 36,2\% de la población adulta, un aumento significativo desde la última medición, rompiendo una tendencia a la baja de cuatro años lo que significa que el ecuador 3,6 millones de habitantes estuvo involucrado con una actividad de emprendimiento; el informe también indica que hay más emprendimientos nacientes que los ya formados, según la revista Líderes del 20 de julio del 2020 en la cual también citan que en el Ecuador que la tasa de emprendimientos por necesidad va en incremento.

Para ser emprendedor no solo basta tener ideas; hay que validarlas, ponerlas en práctica. Para crear un negocio se debe tener un motor como motivación, ser visionario, buscar ideas, prestar atención a la realidad, el contexto, el mercado entre otros.

Actuaciones de un emprendedor; adaptación propia de Sánchez (30 de noviembre de 20I5)

- Localiza nuevas oportunidades mediante el estudio y análisis del mercado y sus cambios. Así, conoce las actitudes y preferencias del público.

- Reúne recursos de tipo financiero, tecnológico o humano para poder acometer el nuevo proyecto.

- Pone en marcha el negocio con determinación y afrontando su posición de responsabilidad, teniendo siempre presente una capacidad de innovación y originalidad 
Debemos entender que para ser emprendedor debemos tener una visión global afuera y adentro; en el primer caso debemos revisar: nuestras aspiraciones, nuestros recursos, nuestras capacidades, nuestros requerimientos, actitudes, debilidades, necesidades y ¿Cómo? podríamos utilizar todo esto en tiempos de pandemia. En el segundo caso nuestras ideas deben ser validadas y puestas en acción haciendo un estudio de mercado, utilizando herramientas actuales y tecnológicas aplicando el marketing mix, con estudios exploratorios con compradores, con expertos en los productos y testear utilizando redes sociales.

Según la página negociosstart,com las " 20 herramientas online para hacer un estudio efectivo de mercado" más usadas entre las que se pueden destacar: Google keyword planner, Seo chat, Semrush, BuzzSumo, Social Mention, Fan PageSpeed Insights, Page Karma, Google trends y Google Analytics.

Hasta antes de la pandemia el internet y el e-comerce habían crecido poco, en ese entonces había mucha población que no confiaba en estos medios; los bancos no estaban obligados a tener plataformas para atender a sus usuarios, las tiendas, supermercados, locales de comidas y otros esperaban a sus clientes; hoy muchos que se han negado a cambiar están a punto de quebrar o han cerrado sus puertas.

Según "Espacios (Rodríguez et al. 2020)". Afirma que el Ecuador se encuentra en quinto lugar en América Latina ventas anuales de $\$ 800.000 .000$, que las cifras globales del comercio electrónico son difíciles de cuantificar para un mercado pequeño como el nuestro, una de las Mipymes con éxito es De Prati que antes de la pandemia sus ventas electrónicas eran del 3\% y tuvieron un incremento del 50\%. Funky Fish paso del $10 \%$ al $15 \%$ y que 13 de cada 100 compradores comenzaron a comprar de una manera digital con WhatsApp con $49 \%$ y delivery con $44 \%$, este último es resultado de un estudio realizado a 1125 personas.

En el mundo post pandemia hay mucha tarea que hacer, hay que reinventarnos para reactivar la economía, generar nuevos modelos de negocios, la adaptación de lo tradicional es una obligación que todo emprendedor debe resolver. La educación también ha cambiado, los cursos on-line, a distancia, las plataformas educativas como Udemy, Tutellus, Hotmart, Zoom, entre muchas otras aprovechan lo que en otros tiempos no era muy rentable, generando ingresos extras; los entretenimientos y las plataformas aumentaron significativamente como Netflix, Paypal, Facebook, Microsoft, Amazon entre otras.

Statista (7 de abril de 2020) en su artículo "Las apps que han despertado más interés en el último mes" habla sobre los hábitos del consumo y el descubrimiento de nuevas aficiones y analizando los gráficos de tendencias de Glimpse afirma que en abril del 2020 en confinamiento Netflix Party creció el interés por visualizarlo en $9.550 \%$, Microsoft Teams 1.100\%, Zoom 875\%, Skype 550\%.

Según datos de la Superintendencia de Compañías, en ese tiempo se registraron 28I nuevos negocios en el país. De ese grupo, 89 establecimientos se ubicaron en ese segmento. La mayoría se concentró en las provincias más pobladas como Pichincha, Guayas y Manabí. 
También ocurrió en Azuay, El Oro y Santo Domingo. En este último cantón, las tiendas repletas de productos de primera necesidad son un nuevo nicho de mercado. Decenas de emprendedores adecuaron y crearon estos locales". El Comercio (6 de junio de 2020)

El cambio gracias a la pandemia fue impresionante para los negocios, la gente se unió y formaron grupos de WhatsApp para ayudarse, regreso el trueque, el "a cambio de...", Marketplace entre otros se convirtieron en herramientas necesarias y los grupos con intereses de negocio crecieron, la oferta se disparó y comenzó la guerra de precios, los negociantes y emprendedores tuvieron que aprender a las buenas o las malas a manejar herramientas tecnológicas y plataformas como Mercado libre, Remate al martillo, Patio tuerca, OLX, etc.

El emprendedor está obligado a capacitarse más y la actitud ya no alcanza, la aptitud en medios y herramientas tecnológicas, marketing y especialidad de su producto son base para comenzar un emprendimiento.

La nueva realidad no ha cambiado la esencia del emprendedor solo lo ha mejorado y exigido nuevas competencias, nuevos conocimientos, nuevos saberes en base a los nuevos hábitos de consumo de nuestro mercado potencial.

Según la plataforma de tendencias Glimpse durante abril del 2020 las tendencias de interés en cuanto a productos y servicios de los internautas mostro que el interés por levadura de pan creció en $1392 \%$, el corte de pelo en casa $662 \%$, pesas $611 \%$, iglesia online $360 \%$. Statista (2020)

\section{Modelos, planes y enfoques}

Hay que adaptar los emprendimientos a la realidad que nos deja la pandemia generando nuevos modelos y planes de negocios. "Un modelo de negocio describe las bases sobre las que una empresa crea, proporciona y capta valor" (Osterwalder, y Pigneur, 201 I, p.I4).

Mientras que el plan de negocios seria cómo se va a ejecutar esa idea para que funcione, en si es un mecanismo detallado mediante el cual se deberán generar los beneficios esperados según. (Pazmiño, s.f.).

Un aporte muy significativo al emprendimiento es lo que hacen en 1990, W. Chan Kim y Renée Mauborgne, con un concepto clave en su libro "La estrategia del océano azul", planteaban dejar de lado la competencia entre las organizaciones, para dar cabida a la ampliación de los mercados con ideas innovadoras en mercados saturados. Por esta razón hay que crear negocios disruptivos y aprovechar con innovación, que se generen nuevos patrones de consumo, de oferta y demanda, nuevas tendencias como lo hizo Wal-Mart o Alibaba en períodos anteriores; Se debe que crear negocios para dar frescura a mercados ya saturados y que con el efecto económico COVID más la proliferación de negocios informales genera un aparente caos.

Propuesta de valor: La propuesta de valor es la clave para adquirir la ventaja competitiva y llegar a captar a los clientes superando del todo sus necesidades.

Es la solución a los problemas de los segmentos de mercado a quienes se dirige 
la empresa ofreciendo un conjunto de productos y servicios los cuales cuentan con características y beneficios generadores de valor. (Casas, y Poveda, 2017). "La IMN (Innovación del modelo de negocio) ayuda a planear mejor las innovaciones disruptivas, motivadas particularmente por la tecnología y que requieren de nuevas competencias estratégicas" (Mejia-Trejo, y Sánchez-Guitierrez, 20I4).

Más que construir un negocio fuerte, la tendencia que marca la nueva normalidad es fomentar un modelo empresarial flexible (para el jefe y sus trabajadores) que sepa amoldarse en poco tiempo tanto a las necesidades del mercado como a la de sus empleados o clientes. (Núñez, 17 de septiembre de 2020).

Otro concepto muy importante para generar nuestros nuevos elementos de emprendimiento es la economía colaborativa "Es relevante porque la economía colaborativa se está utilizando actualmente para la creación de nuevos modelos de negocios innovadores y de alcance global, basados en plataformas digitales" (Córdova Ticona, 2019, p.4).

El término "economía colaborativa" proviene de la expresión inglesa sharing economy, y fue divulgada separadamente por Lisa Gansky y Rachel Bootsman con Roo Rogers en 2010, para referirse a aquellas actividades realizadas sobre redes distribuidas de individuos y comunidades conectados que transforma la forma de producir, consumir, financiarse y aprender y lo contrapone a la tradicional basada en instituciones centralizadas" (Gómez-Álvarez Díaz, y Morales Sánchez, 2018, p. 17).

No se puede hablar de nuevos emprendimientos sin dejar de lado el concepto de: adaptación al cambio y no hay momento más oportuno para aplicarlo.

"En estos tiempos, la importancia de la adaptación al cambio cobra mayor relevancia, no sobreviven los más fuertes, o los más listos, sino aquellos que saben adaptarse mejor a los cambios. Por este motivo, en tiempos especialmente interesantes, lo mejor es ser capaz de adaptarnos de la manera más flexible posible a los cambios presentes y futuros" (Fernández 202I)

Un emprendedor aprovecha oportunidades, para hacer lo que siempre se hacía, pero de manera más ágil, ve los cambios donde otros quieren quedarse; la mejora los procesos, dinamiza y crea nuevos mejorados emprendimientos.

Por último, el Benchmarking con enfoque en los negocios exitosos que están creciendo, que se mantienen estables, que han cambiado sus estrategias para llegar a sus clientes y prospectarse en nuevos nichos; aprovechando las oportunidades que deja la pandemia. "El benchmarking es un proceso continuo por el cual se toma como referencia los productos, servicios o procesos de trabajo de las empresas líderes, para compararlos con los de tu propia empresa y posteriormente realizar mejoras e implementarlas". (Espinoza 2017).

\section{Ideas, oportunidades y adaptaciones de emprendimiento}

Por lo expuesto anteriormente a continuación, se presenta varios ejemplos reales que fueron acomodados y pueden servir para crear o adaptar emprendimientos innovadores 
como para que el lector adapte las ideas a una personalizada sin perjuicio a las reglas y estrategias formales de creación de un negocio.

I.- Gimnasios

ON Spinning On-line Red bike studios. Es una empresa que nació en los 80 impulsada por el estilo del ejercicio en la bicicleta y que a raíz de la pandemia tuvo que cerrar y reinventarse según lo afirma Albert Ghitis dueño del gimnasio. Otro ejemplo es Gloves con plataforma que brinda servicios de Fitness, cursos de gimnasia, alquiler de equipos, etc.

"De acuerdo con las estadísticas que manejan la Federación de Físico Culturismo y la Agrupación de Gimnasios, aproximadamente 1.600 .000 personas acudían a los gimnasios cada mes". El Comercio (03 de julio del 2020).

Con 1.600 .000 personas como mercado potencial un emprendedor debe aprovechar no solo lo referente al gimnasio, sino también en ser recursivo y también mirar al baile como producto viable.

Otros productos que podría tomar en cuenta son: alquiler de equipos y máquinas de gimnasio, cursos y seminarios online para mejorar y mantener el estado físico en encierros.

2.- Locales de comidas

Bien es cierto que la comida a domicilio ya existía antes de la pandemia, con la llegada del Covid muchos crecieron exponencialmente con el Delivery o el Take Away. Existen ejemplos gastronómicos a nivel mundial como Birdcall, Brooklyn Dumpling shop, Gaggan Anand, Bon Duelle Food service vegetales que tienen servicio plataforma online.

Cocinas ocultas o Dark Kitchens es un ejemplo de cómo redes especializadas en preparar comida a domicilio y entregar ágilmente.

"Las cocinas fantasmas" crecen en Quito ejemplos como Aguacate House, Pumpkin, Burger lab entre otras son algunas dar Kitchens que nacieron y se expandieron en tiempos de crisis sanitaria que son restaurates digitales y se manejan con servicios de delivery como Rappi, Uber Eats y Glovo según lo afirma Expreso (9 de diciembre del 2020).

La comida siempre fue y será un buen negocio, solo se ha modernizado y cambiado la estrategia de llegar a clientes otros ejemplos de emprendimientos que se podrían hacer son:

- Delivery gourmet (asesor de cocina vía telemática)

- $\quad$ Food Truck en barrios (el camión de helados, de hamburguesas y hot dog)

- Cursos de comida vegana, repostería vegana on-line se le puede complementar con venta cruzada de productos como la leche de soya, el gluten, alimentos orgánicos, etc.

- Vegan Fodds, vegan diet, delicatesen vegano a domicilio.

- Cursos para la fabricación de yogurt, bebidas, embutidos, helados, conservas, condimentos, etc. vía on-line.

- $\quad$ Easy Order plataforma ayuda en la elección de lugares de comida. 


\section{3.- Cuidado de mascotas}

"Al enfocarse en el mercado de mascotas, Packaged Facts predice un gran salto en las ventas online en el 2020 , al $31 \%$ de las ventas totales de productos para mascotas, en comparación con la predicción previa a la pandemia del 24 por ciento, y el comercio electrónico continuará creciendo hasta 2024" Estrudend (6 de julio del 2020)

Según el portal Primicias (20 de septiembre del 2020) en cifras del Servicio de Rentas Internas muestran que las ventas del segmento de alimentos para animales crecieron $7,4 \%$ entre enero y junio de 2020 , con respecto al mismo periodo en 2019 además casi 60 millones corresponden a comida para mascotas de los cuales la empresa Barfuss en Guayaquil invirtió 100.000 dólares en una planta de producción. Mas las proyecciones para el 2022 prevén un aumento de 2000 millones en la venta según el mismo artículo. Gabrica, Laika especializados en comidas para mascotas a domicilio cuentan con plataforma 24/7 y red de veterinarios; Puppis cuenta con consultas por video llamada para sus clientes.

Otros emprendimientos para mascotas que se podrían realizan en pandemia podrían ser:

- $\quad$ Limpieza y peinado de mascotas a domicilio.

- Veterinario a domicilio (Hospital animal en camino).

- Escuela para mascotas.

- $\quad$ Entrega de juguetes y comida para mascotas a domicilio.

- $\quad$ artículos y ropa para mascotas a domicilio.

4.- Productos de limpieza, desinfección y bioseguridad.

Esta parte de la industria de la limpieza e higiene que más se ha desarrollado a raíz de la pandemia con la venta del alcohol, gel, amonio cuaternario de quinta generación, mascarillas con diseños personalizados, protectores faciales, trajes de bioseguridad hasta colgantes protectores de Covid entre muchos otros.

Por esta misma razón la oferta a crecido de manera sustancial con un desborde de los emprendimientos de productos de limpieza. Para Glimpse (s.f.) revisado el 15 de mayo del 202I plataforma de tendencias no obvias afectadas por el coronavirus; el papel higiénico subió un $+146 \%$, los desinfectantes $+388 \%$, toallas de papel $+306 \%$.

La corporación Azende dueña del licor Zhumir fue una de las empresas ecuatorianas que aprovecho la oportunidad creando el alcohol antiséptico Alcomax a partir del alcohol etílico según el diario el universo (20 de marzo del 2020)

Freecovid. Es una empresa que brinda asesoría completa en protocolos de bioseguridad mientras que HackThepandemic. Manufactura mascarillas de bioseguridad con tecnología 3D.

Otros emprendimientos en los que podrían atraer serian:

- Máquinas para desinfección Ultra violeta, ozonificador, alfombras químicas.

- $\quad$ Servicio de limpieza y desinfección exprés a domicilio.

- Máquinas, portales y túneles de desinfección

- Venta de equipos y uniformes de bioseguridad.

- $\quad$ Lavado, limpieza y desinfección de autos. 
- $\quad$ Limpieza automática y desinfección de motos y bicicletas a domicilio.

- $\quad$ Entrega de productos de aseo y limpieza (kits) a domicilio.

5.- Cuidado de niños.

“"Los niños más pequeños necesitan un poco de entretenimiento, y si los padres están trabajando, no siempre pueden darles el tiempo que necesitan", le cuenta a la BBC Richard Conway, fundador de Childcare.co.uk, la mayor plataforma online de cuidado infantil en Reino Unido". BBC News (28 de marzo del 2020).

Los Pequeños también se estresan pues dejaron su habitual forma de vida como el de jugar y ver a sus compañeros, la forma lúdica de que aprendan y se entretengan mientras los padres trabajan puede ser una idea de negocio, como lo hace Champion Games. Crea Juegos de videos y Mobbyt Considerado el YouTube de los videos educativos.

- Driven y Listen es una aplicación web en la que se puede viajar sin salir de casa.

- $\quad$ Clases especializadas para niños online.

- Video conferencias para el entretenimiento y aprendizaje de niños.

- Cursos y talleres online.

6.- Venta de Utensilios plásticos de fácil biodegradación, fundas, papel, caucho y otros productos llamados verdes; vía plataforma.

"François de Bie, presidente de European Bioplastics, dijo: Nuestra industria ha superado con éxito los desafíos planteados por la pandemia Covid-19, y las perspectivas también son prometedoras, ya que se prevé que el mercado global crezca un $36 \%$ durante los próximos 5 años. En este sentido, se espera que la capacidad de producción mundial de bioplásticos pase de las cerca de 2,I millones de toneladas de este año 2020, a los 2,8 millones de toneladas en 2025". (MundoPlast 2020)

El movimiento verde está en crecimiento, la concientización por el medio ambiente, por generar consumo responsable lo que ha llevado a una revolución en temas de productos eco amigables y orgánicos; las empresas formaron el marketing ecológico para acercarse a este mercado demostrando que sus productos son amigables y sustentables.

- $\quad$ E-comerce de productos ecológicos.

- $\quad$ Envases sustentables de bambú, coco, palma para locales de comidas.

- $\quad$ Producción y venta de productos derivados de desperdicios plásticos

- $\quad$ Fundas biodegradables y otros a domicilio.

- $\quad$ Papel e impresiones en base a derivados del platanillo y tintas ecológicas.

- Antigüedades y muebles compra, venta y reparación.

7.- AP Departamento del servicio al cliente.

- Administración de clientes para pequeñas empresas.

- Tele mercadeo para PYMES.

- Servicios de cobranzas.

- Servicios contables, tributarios y asesoría financiera e inversión online. 
- Asesoría en inversiones vía telemática en: depósitos de alto rendimiento (Cedes), ETFS,

- $\quad$ Archivo y digitalización de documentos físicos.

8.- Manufacturas.

- Hágalo Usted (Bisutería de fantasía)

- $\quad$ Etsy Ecuador plataforma "Artículos mágicos llenos de significado que no encontraras en otro lugar"

- Cursos y ventas online para fabricar jabón artesanal, perfumes relajantes y velas decorativas.

- $\quad$ Cursos y talleres de bisutería y manufacturas.

- $\quad$ Compra, arreglo, venta de peluches y muñecos de felpa.

9.- Transporte, camionetas y taxis. Mensajería y correos

- $\quad$ Plataformas como Uber, Cabify, Raptor, Movil Move entre otros.

- $\quad$ Entrega de paquetes por medio de Drones.

- $\quad$ Asociaciones de camionetas y motos con plataforma.

- Monitoreo y seguridad vía satélite.

- Delivery, mensajería exprés, Ali express.

10.- Tiendas de barrio y almacenes.

- $\quad$ Tiendas del barrio 2.0 según Facebook Business (2020).

- $\quad$ Entrega a domicilio.

- $\quad$ Tienda social de compras (Varios artículos de varios vendedores independientes por medio de una plataforma) (Poli Ford, Shop que).

- Venta de moda ética responsable y sostenible.

- $\quad$ Catálogo, pedido virtual y telemarketing.

- Dropshipping (Shopify "tu tienda ecommerce", Tenderati, Fulfillment, Printful entre otras plataformas)

II.-Juguetes para niños.

- Impresoras 3D.

- Juegos de mesa.

- Juegos dinámicos y de entretenimiento.

- $\quad$ Plataformas de juegos y educación.

- Inyectora de juguetes didácticos.

12.-Reciclaje.

- Reciclaje y reutilización de llantas para muebles, adornos, maceteros, ropa, juguetes.

- $\quad$ Compra y renovación de muebles por medio de Marketplace.

- Compra de aceite de cocina usado

- Compra, venta y reparación de herramientas usadas. 
- $\quad$ Compra y venta de ropa, Uniformes escolares y disfraces en perfecto estado.

- Electrodomésticos usados compra y venta por plataformas como mercado libre o Marketplace.

- Tendencia retro como Discos de acetato, electrónica antigua, artículos antiguos.

13.- Departamento de marketing, publicidad y empresas en general.

Venta sin contacto "Entrega con drones y con música"

Asesores en marketing digital, creador de contenidos, comunity manager, brandy personal, social marketing, podcast, software, páginas web, automatizaciones, digitalización de PYMES y microempresas, digitalización de documentos, ferias y exposiciones virtuales, digitalización de espacios públicos.

- $\quad$ Parapentex Estudios Big data.

- Micro SAS software de servicio específico.

- RepScan retira en internet contenido negativo contra cualquier persona.

- Citrix soluciones centradas en las personas que facilita una mejor forma de trabajar al ofrecer aplicaciones y datos seguros en cualquier dispositivo.

- Zendesx software de soporte al cliente, adaptada a empresas grandes y pequeñas.

14.- AP Cursos y seminarios y otros.

- Match plataforma que reúne personas solteras

- Terapias online para llevar la cuarentena.

- $\quad$ Coach Teacher academia digital (ofrecer cursos de profesionales que tengan mucho conocimiento digital)

- $\quad$ Fiestas y conciertos virtuales.

- Influencer marketing.

- Dotar de tecnología a sectores rurales

- CubArgentina escuela de baile virtual.

- Teilu plataforma inclusiva considerado el Netflix inclusivo.

- Telemedicina.

- $\quad$ Consultoría legal en línea

- Sotfware de Retargeting (recordar a nuestros clientes que estamos ahí), chatbot como regreso de información al cliente, Landing page (página de aterrizaje o destino) Typeform nos sirve para realizar cuestionarios personalizados, video marketer, entre otros.

- $\quad$ ON E-bay Portal que funciona como tienda de subastas online de todo producto.

- Reacomodación de ambientes para teletrabajo 
Todavía el cambio es la única constante, la adaptación como recurso, mejorar la economía es obligatorio y la necesidad por generar empleos es grande; el emprendedor debe ajustarse, presentar soluciones de alguna manera apalancarse, adaptase y crecer, se puede y se debe emprender en tiempos de pandemia.

La tecnología juega un papel realmente impórtate e impresionante, el internet como servicio básico, la telemática, las redes sociales, el comercio electrónico, las plataformas, la adaptación de los sistemas de trabajo y el teletrabajo entre otros.

En el Ecuador los emprendimientos formales todavía están en crecimiento, se puede invertir de una manera diferente con otra perspectiva y dejar de ver la pandemia como una amenaza. En el mercado global los negocios han cambiado y muchos se quedarán así en post pandemia, porque el cliente ya se acostumbró al nuevo servicio.

La pandemia creo nuevos nichos de mercado, el emprendedor debe moverse y acomodarse de una manera más rápida, en función de las tendencias, la globalización es acelerada, se vale duplicar modelos, estrategias y adaptarlas a nuestro medio.

\section{DISCUSIÓN}

En Ecuador el desempleo es la causa y motivación principal para buscar emprendimientos; el país tiene cifras altas en crecimiento emprendedor, pero la falta de adaptación al cambio y la pandemia fue la razón principal de cierre de emprendimientos, según el INEC para septiembre del 2020 el 48,6\% de personas con empleo se encuentran en el sector informal. En el País si es factible hacer emprendimiento y según datos del GEM el 61,1\% de negocios y emprendimientos aumentaron sus esfuerzos en marketing, el $57,5 \%$ ofrecieron nuevos servicios y productos y el 5 I,3\% apuntan a las ventas online; queda demostrado que existen emprendimientos que pese al crisis siguen creciendo o se han mantenido; después de hacer cambios en el modelo de negocio, hacer adecuaciones y adaptaciones.

Entonces va a depender de la adaptación, de la inventiva, de la dinámica de modelos y estrategias para que el emprendedor pueda reactivar su economía.

Es difícil cuantificar el valor real total de los emprendimientos informales creados en pandemia; de esas personas que viven del día a día, que salen a la calle a ofrecer cualquier producto con todos los peligros que esto atrae; queda la presunción que ial aumentar incentivos al emprendedor formal se reducirá el nivel de personas sin trabajo adecuado y en la calle?

\section{REFERENCIAS}

Asamblea Nacional de la Republica de Ecuador (2020). Ley Orgánica de emprendimiento e innovación. Suplemento del Registro Oficial No. 15I, 28 de febrero 2020. Quito: Asamblea Nacional de la Republica de Ecuador.

Banco mundial (5 de enero 202I). Una recuperación moderada, con daños que reparar. Banco mundial. https://www.bancomundial.org/es/news/feature/2021/0I/05/global-economicprospects 
Casas, W., y Poveda, J. (2017). Modelo de negocio para validar el emprendimiento "Verduritas" en la ciudad de Bogotá (Tesis de Grado). Universidad Libre, Bogotá D.C., Colombia.

Celis Maya, J. S. (20 de abril de 2020). 7 tipos De Negocios Que Se Van A BENEFICIAR Permanentemente De La Pandemia [Vídeo]. YouTube. https://www.youtube.com/watch?v=cl 2FdiV7OOY

Clavellina Miller, J. L. (2020). Posibles efectos del Coronavirus en la economía mundial. Instituto Belisario Domínguez, (75), I-8.

Conexión ESAN (28 de febrero de 2018). ¿En qué consisten y cuál es la diferencia entre océanos rojos y azules? Apuntes empresariales. Economía. Recuperado de https://www.esan.edu.pe/apuntes-empresariales/2018/02/en-que-consisten-y-cuales-la-diferencia-entre-oceanos-rojos-y-

azules/\#: :text=El\%20mundo\%20de\%20los\%20negocios,la\%20competencia\%20en tre\%20las\%20empresas.\&text=Por\%20el\%20contrario\%2C\%20el\%20oc\%C3\%A9a no,mercado\%20haciendo\%20irrelevante\%20la\%20competencia

Córdova Ticona, H. (2019). Economía colaborativa: ¿Transformará la sociedad? (Tesis de Grado). Universidad Peruana de Ciencias Aplicadas, Lima, Perú.

Crea Y Transforma (II de enero de 202I). Las 17 MEJORES INVERSIONES en el 2021 Cómo invertir con poco dinero [Vídeo]. YouTube. https://www.youtube.com/watch?v=j8sM-ijxFSg

Dabián, C. (I 3 de noviembre de 2018). I0I NEGOCIOS RENTABLES PARA INICIAR DESDE CASA - César Dabián [Vídeo]. https://www.youtube.com/watch?v=Eog93lsM4Q।

El Comercio (6 de junio de 2020). La emergencia sanitaria en Ecuador sirvió para emprender y reacondicionar. El Comercio. Recuperado de https://www.elcomercio.com/actualidad/ecuador/emergencia-sanitaria-ecuadoremprendimientos-negocios.html

El comercio (3 de Julio de 2020) Dueños de gimnasios anuncian una marcha para el 8 de julio del 2020. El Comercio recuperado de https://www.elcomercio.com/tendencias/salud/duenos-gimnasios-marcha-quitocovid I 9.html

Espinosa, R. (s.f.). Benchmarking: qué es, tipos, etapas y ejemplos. Recuperado de https://robertoespinosa.es/2017/05/13/benchmarking-que-es-tipos-ejemplos

Estruded Internacional Magazine. (6 de julio del 2020) ¿Cómo se Vislumbra la Industria de Mascotas después de los Subsidios de la Pandemia COVID-19? Recuperado de

https://allextruded.com/entrada/como-se-vislumbra-la-industria-de-mascotas-despuesde-los-subsidios-de-la-pandemia-covid-19-22638

Expósito, A., Fernández-Serrano, J., y Velasco, F. (2017). Crecimiento económico, pobreza y desigualdad: Un análisis de eficiencia para américa latina en el siglo XXI. Revista de Economía Mundial, (47), II7-138.

Expreso ( 9 de diciembre del 2020) Las cocinas fantasmas crecen en Quito durante la pandemia. Expreso recuperado de 
https://www.expreso.ec/quito/cocinas-fantasma-crecen-pandemia-95028.html Fernández, A. (s.f.). Adaptación Al Cambio En Tiempos De Covid - 19. Recuperado de 19/ https://www.auraquantic.com/es/adaptacion-al-cambio-en-tiempos-del-covid-

Gómez-Álvarez Díaz, R., y Morales Sánchez, R. (2018). Principios ontológicos de la economía colaborativa verdadera. En R. Gómez-Álvarez Díaz, D. Patiño Rodríguez, J.J. Plaza Angulo (Ed.), Economía colaborativa... ¿De verdad? (Pp. I5-4I). Murcia: Laborum

GLIMPS (s.f.). Rastreador De Impacto Del Consumidor COVID-19. Recuperado de https://meetglimpse.com/covid I $9 /$

Güemes, A. (20l8). El emprendimiento y los jóvenes. Dimensiones psico-sociales. México: Plaza y Valdés S.A. de C.V.

IB Training Center (3 de agosto de 2020). 37 de 150 emprendimientos consultados cerraron en pandemia; la mayoría se concentra en Guayaquil. Recuperado de https://ibtrainingcenter.com/37-de-I50-emprendimientos-consultados-cerraronen-pandemia-la-mayoria-se-concentra-en-guayaquil/

Lasio, V., Amaya, A., Zambrano, J., y Ordeñana, X. (2020). Global Entrepreneurship Monitor Ecuador 2019/2020. Ecuador: ESPAE, Escuela de Negocios de la ESPOL.

Martínez, C., y Rubio Bañón, A. (2020). Emprendimiento en épocas de crisis: Un análisis exploratorio de los efectos de la COVID-19. Asociación Española de Contabilidad y Administración de Empresas - AECA, 4(2), 53-66. Doi: 10.26784/sbir.v4i2.279

Mejia-Trejo, J., y Sánchez-Guitierrez, J. (20l4). Factores determinantes de la innovación del modelo de negocios de la creación de ventaja competitiva. Revista del Centro de Investigación Universidad La Salle, I I (42), I05-I28.

MundoPlast (2020). Larga vida al mercado mundial de bio-plásticos. Recuperado de https://mundoplast.com/mercado-mundial-bioplasticos-2020/

Negocio Start (s.f.). 20 herramientas online para hacer un efectivo estudio de mercado. Recuperado de https://negociostart.com/20-herramientas-online-para-hacer-unefectivo-estudio-de-mercado/

Núñez, J. (17 de septiembre de 2020). ¿Qué tipos de negocio sobrevivirán al coronavirus? El País. Recuperado de https://elpais.com/economia/2020/08/20/nuevos_tiempos/I5979I99II_259535.ht $\mathrm{ml}$

Osterwalder, A., y Pigneur, Y. (20II). Generación de modelos de negocio. Un manual para visionarios, revolucionarios y retadores. Barcelona, España: Centro Libros PAPF, S. L. U.

Pagés, S. (2 de julio de 2020). Emprendimiento en tiempos de pandemia. Recuperado de https://www.cajasietecontunegocio.com/temas/direccion-yemprendeduria/item/emprendimiento-en-tiempos-de-pandemia-covid I 9

Parra Alviz, M., Rubio Guerrero, G., y López Posada, L. M. (2019). Emprendimiento y creatividad. Aspectos esenciales para crear empresa. España: ECOE ediciones. 
Pazmiño, A. (s.f.). La diferencia entre el plan de negocios y el modelo de negocios [Mensaje en un blog]. Recuperado de https://bold.com.ec/plan-de-negocios/

Real Academia Española (s.f.). Emprender. En Diccionario de la lengua española. Recuperado en 23 de mayo de 202I, de https://dle.rae.es/emprender

Rodríguez, k., Ortiz, O., Quiroz, A., Parrales, M., (2020) El E-comerce y las Mipymes en tiempos de Covid - 19, Volumen 4I, Pág. II3 - II7- conclusiones. ISSN 0798-1015. DOI: 10.48082/espacios-a20v4 In42p09.

Recuperado de https://revistaespacios.com/a20v4In42/a20v4In42p09.pdf

Sánchez, J. (30 de noviembre de 2015). Emprendedor. Recuperado de https://economipedia.com/definiciones/emprendedor.html

Statista (7 de abril de 2020). Las apps que han despertado más interés en el último mes [Mensaje en un blog]. Recuperado de https://es.statista.com/grafico/2 I 36 //variacion-del-interes-de-los-internautas-enel-ultimo-mes-en-una-seleccion-de-apps/

Statista ( 9 de abril de 2020). Los productos más populares durante el coronavirus [Mensaje en un blog]. Recuperado de https://es.statista.com/grafico/21359/productos-mas-demandados-coronavirus/

Sulbarán Lovera, P. (8 de mayo de 2020). Economía y coronavirus: 7 emprendimientos de Latinoamérica que se reinventaron en medio de la pandemia y están prosperando. BBC News Mundo. Recuperado de https://www.bbc.com/mundo/noticias-america-latina-52495847

Torres, W. (27 de febrero de 202I). La OIT proyecta que la pobreza en Ecuador subirá al $32 \%$. Primicias. Recuperado de https://www.primicias.ec/noticias/economia/oitpobreza-ecuador-subira-pandemia/ 